Nonlinear Processes in Geophysics (2003) 10: 351-361

Nonlinear Processes

in Geophysics

(C) European Geosciences Union 2003

\title{
On the predominance of oblique disturbances in the supersonic shear flow instability of the geomagnetic tail boundary
}

\author{
V. V. Mishin \\ Institute of Solar-Terrestrial Physics Russian Academy of Sciences, P.O. Box 4026, Irkutsk 664033, Russia
}

Received: 4 July 2001 - Revised: 6 May 2002 - Accepted: 23 May 2002

\begin{abstract}
A study is made of the influence of the longitudinal magnetic field and density inhomogeneity on the supersonic shear flow instability at the magnetospheric tail boundary. It is shown that the most unstable are slow oblique (3D) disturbances, with a phase velocity approaching at a sufficiently large angle (with respect to the flow direction) the magnetosonic velocity. Their growth rate and spectral width are much larger than those of the usually considered longitudinal (2D) supersonic disturbances. The magnetic field reduces the compressibility effect and, unlike the subsonic case, has a noticeable destabilizing effect on the excitation of oblique disturbances.
\end{abstract}

\section{Introduction}

The shear flow instability at the magnetospheric boundary is known in the geophysical literature as the Kelvin-Helmholtz instability (K-HI). This instability is considered as the key excitation mechanism for magnetopause oscillations and associated geomagnetic pulsations (Dungey, 1955; Moskvin and Frank-Kamenetsky, 1967; Southwood, 1974; Kovner et al., 1977). Besides, the K-HI can play an important role in the solar wind - magnetosphere interaction by ensuring the momentum transfer to the magnetosphere and forming diffuse boundary layers (Boller and Stolov, 1970; Mishin, 1979; Southwood, 1979). The K-HI theory was developed mainly for subsonic disturbances which are dominant at the dayside magnetopause and at the near geomagnetic tail boundary $\left(|x|<50 R_{E}\right)$. In this region, the magnetosheath magnetic field plays a stabilizing role (Boller and Stolov, 1970), increasing with the azimuthal interplanetary magnetic field (IMF). This is in a qualitative agreement with the behavior of daytime geomagnetic Pc3 and Pc4 pulsations (Mishin, 1981). On the other hand, at the far tail boundary, the flow velocity is a hypersonic and hyper-Alfvenic one. Because of the strong compressibility, it is commonly believed that the K-

Correspondence to: V. V. Mishin (vladm@iszf.irk.ru)
HI plays only a minor role (if any) in this case (Miura, 1990, 1992). However, the observed broad boundary layers, longperiod (10 $\mathrm{min}$ ) boundary oscillations (Sibeck et al., 1987), and high-latitude geomagnetic pulsations (Pudovkin et al., 1976) are hardly explainable, unless the K-HI develops at the far tail boundary. The objective of this paper is to demonstrate that this is actually the case.

Previous instability analyses use essentially the tangential discontinuity approximation (Landau, 1944), just with which the name "K-HI" is associated (Sen, 1964; Parker, 1964; McKenzie, 1970; Ershkovich and Nusinov, 1972; Southwood, 1979; Pu and Kivelson, 1983; Kivelson and Chen, 1995). Within this approximation, the stabilizing influence of the magnetic field is analytically described when the velocity difference is subsonic. However, this longwavelength approximation is not applicable for the most unstable short-wavelength disturbances (Chandrasekhar, 1962; Moskvin and Frank-Kamenetsky, 1967), as well as for the supersonic velocity difference (Landau, 1944).

In this paper, the shear flow instability is studied beyond the tangential discontinuity approximation. The boundary is approximated by a diffusive shear layer of thickness $D=2 d$ near the plane $z=0$. On the layer there occur variations of the flow velocity $\mathbf{v}_{0}(z)$, density $\rho_{0}(z)$, temperature, and magnetic field $\mathbf{B}_{0}(z)$. These are assumed to vary along the axis $z$, and the vectors $\mathbf{v}_{0}(z)$ and $\mathbf{B}_{0}(z)$ are collinear to the plane $z=0$. For the sake of simplicity, it is assumed that only the magnitudes of the velocity and magnetic field vary with $z$, while their direction remains unchanged.

The dispersion properties of the shear flow instability with a smoothly-varying velocity profile $\left(\mathbf{v}_{0}(z) \propto \tanh (z)\right)$ have been thoroughly studied within the approximation of an incompressible medium (Chandrasekhar, 1962; Michalke, 1964; Moskvin and Frank-Kamenetsky, 1967; Chandra, 1973; Walker, 1981; Morozov and Mishin, 1981; Miura, 1982). This approximation holds if the velocity difference on the shear layer $\Delta v=v_{I I}-v_{I}$ is small compared to the magnetosonic velocity $c_{m}=\sqrt{c_{s}^{2}+a^{2}}$, i.e. when $M_{m s}=$ $\triangle v /\left(2 c_{m}\right) \ll 1$. Here $c_{s}^{2}=\gamma P_{0} / \rho_{0}$, and $a^{2}=B_{0}^{2} /\left(4 \pi \rho_{0}\right)$, 
respectively, are the sound and Alfven velocities squared; $P_{0}$ is thermal pressure; and the indices I and II correspond to the magnetosphere and the magnetosheath, respectively. Subsonic disturbances are surface ones, as their amplitude decreases rapidly with distance away from the shear layer. With its longitudinal (with respect to the flow direction $\mathbf{v}_{0}$ ) component $\mathbf{B}_{0}=\mathbf{B}_{0 \|}$, the magnetic field reduces the maximum growth rate of the generated disturbances and narrows their frequency range (Chandra, 1973; Miura, 1982; Mishin and Morozov, 1983; Shukhman, 1998). A similar effect is exerted by the compressibility of the medium (Blumen, 1970; Blumen et al., 1975).

The density gradient shifts the maximum growth rate $\gamma=$ $\operatorname{Im}(\omega)$, and the short-wavelength instability boundary $(k d)_{b}$ towards shorter length-scales along the axis $k d$, where $k$ is the wave number (Morozov and Mishin, 1981). Thus the effects of the magnetic component aligned with the flow and of the compressibility are opposite to that of the density inhomogeneity. It turns out that the influence of the compressibility upon the instability is essential at the magnetospheric boundary near the dawn-dusk meridian (Mishin, 1981) where the flow is supersonic $v_{0} / c_{m} \geq 1$. Therefore, when, for analyzing the dispersion properties of the shear flow at the magnetospheric flanks, it is necessary to simultaneously take into account the influence of the following three factors, as follows: the density inhomogeneity, the magnetic field, and the compressibility. Such an analysis was never performed for the magnetopause instability (Sen, 1964; Parker, 1964; Moskvin and Frank-Kamenetsky, 1967; McKenzie, 1970; Ong and Roderick, 1972; Ershkovich and Nusinov, 1972; Southwood, 1979; Walker, 1981; Pu and Kivelson, 1983; Kivelson and Chen, 1995; Miura, 1982, 1990, 1992, 1996, 1999; Farrugia et al., 2000). Apparently, this can distort results of a nonlinear modeling of the shear flow instability (Wu, 1986; Belmont and Chanteur, 1989; Miura, 1990, 1992, 1996, 1999; Shen and Liu, 1999) as well as analysis results on the excitation of waveguide modes (Mann et al., 1999).

\section{On the modification of the algorithm for numerical analysis of the supersonic instability}

We investigated the instability of a supersonic shear flow with a smooth velocity profile given by $v_{0}(z)=u \cdot[1+$ $\tanh (z / d)$ ] about two decades ago (Morozov and Mishin, 1981; Mishin and Morozov, 1983). Those computational results on subsonic disturbances have remained unaltered to date. The position of a maximum of the dispersion curves $\omega(k)$ relative to the axis $\alpha=k d$ was determined sufficiently accurately for the supersonic velocity difference. However, the accuracy of calculations of the absolute values of the growth rate of supersonic disturbances was low, viz. their values were too high. On this basis, the conclusions drawn by Mishin and Morozov (1983) about the predominance of near-sonic disturbances over the value of the growth rate might well appear as not obvious. It is for that reason that this paper carries out a more accurate numerical analysis of the supersonic shear flow instability and of its characteristics on the geomagnetic tail boundary, with due regard for the influence of the inhomogeneities of density and the longitudinal magnetic field.

The density, velocity and magnetic field profiles are specified in the same manner as done in (Mishin and Morozov, 1983):

$\rho(z)=\rho_{00}\{1+\varrho \cdot[1+\tanh (z / L)]\} /(1+\varrho) ;$

$v_{0 x}(z)=u \cdot\{1+\tanh (z / d)\}$

$\mathbf{B}_{0}(z)=\mathbf{b}_{00} \cdot\{1-\delta[1+\tanh (z / h)]\} /(1-\delta)$.

The temperature profile $T(z)$ is obtained upon substituting the expressions (1)-(3) into the equilibrium condition which in the absence of fields of external forces, $g=0$, implies a constancy of total pressure

$P_{0}(z)+\mathbf{B}_{0}(z)^{2} /(8 \pi)=$ const.

When the profiles are specified in Eqs. (1)-(3), the layer of a "sharp" change lies near the plane $z=0$, and the scales of variation of all parameters may not coincide in the general case. Furthermore, by appropriately choosing the amplitudes involved in the expressions (1)-(3), it is possible to approximate sufficiently well the actually observed profiles. For the sake of simplicity, in the subsequent discussion the scale of density and field variation will be considered equal to the scale of velocity variation $L=h=d$.

Upon linearizing the MHD equations for disturbances:

$f_{1}(x, y, z, t) \propto f(z) \mathrm{e}^{1\left(k_{x} x+k_{y} y-\omega t\right)}$

we obtain (Mishin and Morozov, 1983) the following system of differential equations for vertical displacement amplitudes $\xi$ and for a total pressure perturbation $\Pi_{1}=P_{1}+$ $\mathbf{B}_{0} \cdot \mathbf{B}_{1} /(4 \pi)$ :

$\Pi_{1}=\rho_{0} \frac{\Omega^{2}}{\chi^{2}} \xi^{\prime}$

$\Pi_{1}^{\prime}=\rho_{0} \Omega^{2} \xi$

Here $\mathbf{k}=\left\{k_{x}, k_{y}, 0\right\}$ is the wave vector, $\omega$ is the cyclic frequency, the prime ' designates the derivative with respect to the coordinate $z, \Omega^{2}=\tilde{\omega}^{2}-(\mathbf{k} \cdot \mathbf{a})^{2} ; \tilde{\omega}=\omega-\left(\mathbf{k} \cdot \mathbf{v}_{0}\right)$;

$\chi^{2}=k^{2}-\frac{\tilde{\omega}^{4}}{\left[\tilde{\omega}^{2}\left(c_{s}^{2}+a^{2}\right)-(\mathbf{k} \cdot \mathbf{a})^{2} a^{2}\right]}$

The system of Eqs. (4)-(5) with the specified distributions of equilibrium parameters, along with the decrease condition $\lim \left|f_{1}^{\prime}(z)+\chi f_{1}(z)\right| \rightarrow 0$ when $z \rightarrow \infty$, represents a problem of seeking the eigenfunctions $\Pi_{1}, \xi$ and the eigenvalues of $\omega$, which we solved in the following way. The domain of integration of Eqs. (4)-(5) $|z| \leq R$ was chosen such that the condition $R=5 d$ is satisfied. Then, when $|z| \leq R$ the solution of $f_{1}(z)$ must approach in character the solution of 
the problem of the tangential discontinuity (TD) instability, i.e. $f_{1}(z) \propto \exp (-\chi|z|)$, when $\operatorname{Re}(\chi) \geq 0$. Therefore, $f_{1}(z)=f_{0} \exp (-\chi|z|)$ when $z=-R$ was substituted into equations (4)-(5), and the resulting quadratic algebraic equation was used to determine the value of $\chi$ as a function of the complex value of the frequency $\omega$ (the sign of the root of $\chi$ was chosen in accordance with the decrease condition from the boundary).

After that, when $z=-R$, for dimensionless quantities it was assumed that $\tilde{f}_{1}(z=-R)=\varepsilon$ and $\tilde{f}_{1}^{\prime}(z=-R)=$ $\tilde{\chi} \tilde{f}_{1}(z=-R)$ (the symbol $\sim$ designates a dimensionless quantity), and with these initial conditions, Eqs. (4)-(5) were integrated over the real axis $z$ right up to $z=R$. Furthermore, the presence of a finite growth rate is assumed. To avoid the inclusion of singular points in the path of integration at small values of the growth rate $\tilde{\gamma} \rightarrow 0$, it is necessary to keep track of the fulfilment of the smallness condition of the integration step compared with the dimensionless growth rate. Since the trial value of $\omega$ for the specified parameters of the medium is not immediately the eigenvalue of the problem, the quantity $\omega$ was treated as the root of the algebraic equation $\tilde{f}_{1}^{\prime}(z=R)+\tilde{\chi} \tilde{f}_{1}(z=R)=0$.

The values of the short-wavelength instability boundary (parameter $\alpha_{b}$ ), which we calculated following the technique described above, are in good agreement with the exact analytical values: both for the tangential (see Chandrasekhar, 1962; Michalke, 1964; Chandra, 1973) and for the linear profiles (Raleigh, 1894; Moskvin and Frank-Kamenetsky, 1967). Noteworthy is the fundamental importance of our using the condition of a smooth exponential decrease of the amplitude for the supersonic velocity difference. The point here is that the condition for the disturbance amplitude going to zero at the right-hand edge of the computing box leads, when $\Delta v \geq c_{m}$, to the (above-mentioned) too high value of the growth rate. Besides, this could bring about a nonphysical solution such as beatings with a large amplitude a short distance from the boundaries of the computing box and with its abrupt vanishing at the edge of $z=R$.

\section{Instability of longitudinal $\mathbf{k}|| \mathbf{v}_{0}$ disturbances}

Here we consider the instability of longitudinal disturbances ( $\mathbf{k}$ is parallel to the direction common to $\mathbf{v}_{0}$ and $\mathbf{O x}, \mathbf{k}=\mathbf{k}_{\|}$) for the following case: 1) in the absence of the magnetic influence, and in the presence of 2) the transverse $\left(\mathbf{k} \perp \mathbf{B}_{0}\right)$, and 3) the parallel $\left(\mathbf{k} \| \mathbf{B}_{0}\right)$ magnetic field.

3.1 On the instability in the transverse flow case: $\mathbf{v}_{0} \perp \mathbf{B}_{0}$ and its characteristics when $M \geq 1$

From the outset, we discard the influence of the magnetic field: firstly, its pressure will be considered weak, $(\beta=$ $\left.8 \pi P_{0} / B_{0}^{2} \gg 1\right)$, and, secondly, we specify the direction of the magnetic vector to be orthogonal to the vectors $\mathbf{k}$ and $\mathbf{v}_{0}:\left(\mathbf{k}\left\|\mathbf{v}_{0}\right\| \mathbf{O x}, \mathbf{B}_{0} \| \mathbf{O y}\right)$. Results of calculations for longitudinal (with respect to the flow direction) disturbances are

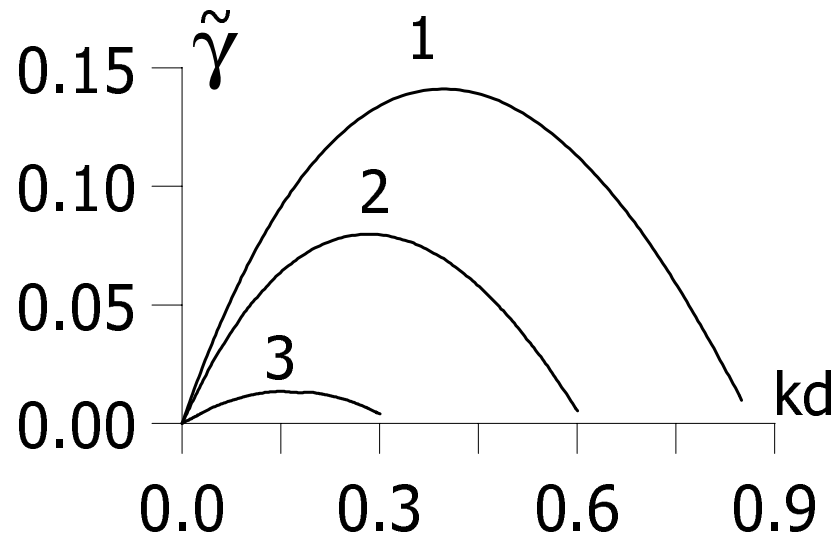

Fig. 1. Influence of the compressibility of the medium on the dependence of the growth rate $\tilde{\gamma}(k d)$ of longitudinal disturbances (k parallel to the flow velocity $\mathbf{v}_{0}$, or $\mathbf{k}=\mathbf{k}_{\|}$), for different values of the $\mathrm{M}$ number. Curves 1, 2 and 3 correspond to the following values of the $\mathrm{M}$ number: $0.5,0.8$, and 1.2.

presented in Fig. 1. The figure plots the dependence of the dimensionless growth rate $\tilde{\gamma}=\gamma d / u$ on the dimensionless wave number $\alpha=k d$.

As is evident from the figure, the compressibility of the medium reduces the growth rate $\tilde{\gamma}_{\max }$ (as would be expected), and displaces it to the left, i.e. toward longwavelengths, both the position of its maximum $\left(\alpha_{\max }\right)$ along the axis $k d$ and the position of the instability boundary $\alpha_{b}$ (i.e. it decreases the instability range). It is easy to see that the influence of the compressibility of the medium (i.e. the variations of the number $M$ ) is similar to the influence of the longitudinal magnetic field when $M<1$ : with an enhancement of the compressibility of the medium, there is a decrease of the maximum growth rate $\tilde{\gamma}_{\text {max }}$, and a narrowing of the range of unstable disturbances $\alpha_{b}$. The values $\alpha_{b}$ of subsonic perturbations (curves 1 and 2) satisfy Howard's semicircle theorem (Blumen, 1970), determining the position of $\alpha_{b}$ on the plane $(\alpha, M): \alpha_{b}^{2}+M^{2}=1$. Furthermore, our computational results for supersonic disturbances are in good agreement with the findings reported in (Blumen et al., 1975; Drazin and Davey, 1977), where in the ordinary hydrodynamics (with no magnetic field) the influence of the compressibility of the medium was studied for the first time. Specifically, the cited references investigated in detail the instability of supersonic shear flows which are stable in relation to longitudinal disturbances within the TD approximation (Landau, 1944).

Blumen (1970) and Blumen et al. (1975) reported the following important characteristics of supersonic disturbances.

Firstly, their growth rate is significantly smaller than that of subsonic disturbances when $M<1$. Thus, as the number $M$ varies from 1 to 2 , the value of the dimensionless growth rate decreases from $\tilde{\gamma}_{\max }=0.035$ (when $\alpha_{\max }=0.15$ ) to $\tilde{\gamma}_{\max }=0.002$ (when $\alpha_{\max }=0.125$ ). Furthermore, with a rapid decrease of the growth rate, the short-wavelength instability boundary is displaced rather weakly- from $\alpha_{b} \approx 0.4$ 
$(M=1)$ to $\alpha_{b} \approx 0.26(M=2)$.

Secondly, it is the quasi-radiative character where the function $\chi$, characterizing the exponential decrease of an amplitude for supersonic disturbances becomes nearly imaginary $\operatorname{Re}(\chi) \rightarrow 0, \mid \chi)|\approx| \operatorname{Im}(\chi) \mid$. In this case, our earlier use of the condition of the eigenfunctions of Eqs. (4) and (5) going to zero at the edge of the counting interval $z=R$, which was valid for subsonic (fast decreasing in $z$ ) disturbances, can be violated when $M \geq 1$.

The above characteristics of supersonic disturbances required for carrying out an adequate analysis and, accordingly, for improving the accuracy of calculation, (1) introducing the double counting accuracy; and (2) achieving a more rigorous fulfilment of the amplitude decrease condition, instead of the (well-satisfied within the approximation of an incompressible medium) requirement for the amplitude becoming zero when $z \rightarrow R$, by analogy with (Blumen, 1970; Blumen et al., 1975; Boller and Stolov, 1970; Chandra, 1973; Chandrasekhar, 1962; Drazin and Davey, 1977), we chose to use the condition of an exponential decrease of the amplitude of eigenfunctions. A modified computational algorithm permitted us to carry out an analysis of the MHD instability of supersonic shear flows with due regard for such important factors as the external magnetic field $\mathbf{B}_{0} \neq 0$, and the inhomogeneities of the density and of the field. These factors were not taken into account in (Blumen et al., 1975; Boller and Stolov, 1970; Chandra, 1973; Chandrasekhar, 1962; Drazin and Davey, 1977) in terms of the ordinary hydrodynamics.

Using results from (Blumen, 1970; Blumen et al., 1975; Boller and Stolov, 1970; Chandra, 1973; Chandrasekhar, 1962; Drazin and Davey, 1977) obtained for $\mathbf{B}_{0}=0$, we now verify the results of our calculations of the MHD instability for the case of an arbitrary (in absolute value $(\beta \simeq 1)$ ), but transverse (in direction) magnetic field. To do so, we take into consideration that in this case the effective "Mach" number decreases. As follows from Eqs. (4)-(6) (see also Sen, 1964; Ong and Roderick, 1972), instead of the number $M=u / c_{s}$, for characterizing the compressibility influence when $\mathbf{B}_{0} \neq 0$, one has to take the "magnetosonic Mach number":

$M_{m s}=M / \sqrt{1+a^{2} / c_{s}^{2}}=M / \sqrt{1+2 /(\Upsilon \beta)}$.

(Here $\Upsilon$ is the polytropic index). The decrease of the compressibility influence by the magnetic field (i.e. actually the enhancement of the instability - the increase of the growth rate and of the instability range) can be accounted for by the fact that the magnetic field increases a total pressure $\Pi_{0}=P_{0}+B_{0}^{2} /(8 \pi)$.

This is easy to verify analytically; when $M \ll 1$, within the TD approximation (which holds when $k d \rightarrow 0$ ), it is possible to show that the decrease of the growth rate $\delta \tilde{\gamma}$ with respect to the usual "incompressible" growth rate $\tilde{\gamma}_{\text {incomp }}$ at the expense of the weak compressibility is $\delta \tilde{\gamma} \approx$ $\left\{u^{2} / c_{m}^{2}\right\} \tilde{\gamma}_{\text {incomp }}$, where the denominator now involves not simply the sound velocity squared but the magnetosonic velocity squared $c_{m}^{2}=c_{s}^{2}+a^{2}$.

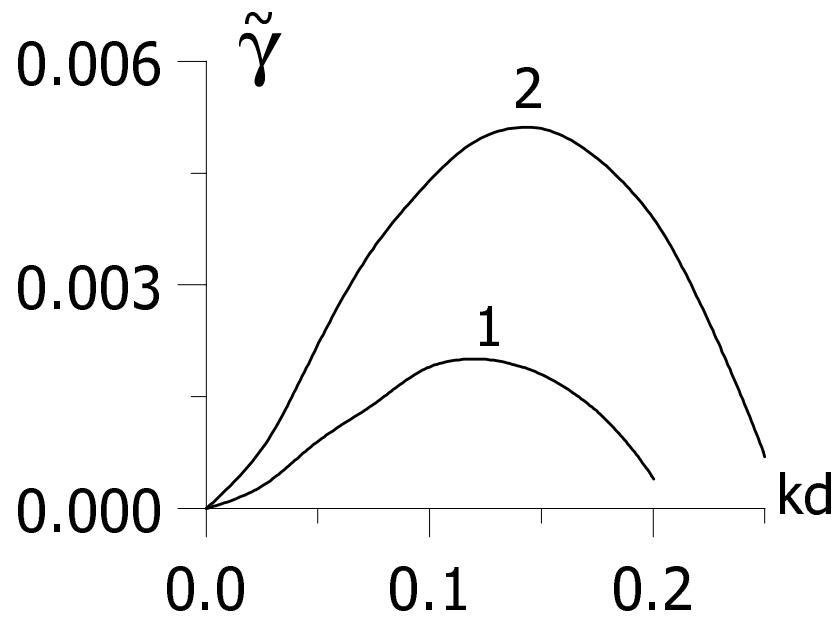

Fig. 2. Influence of the magnetic component $\mathbf{B}_{0 \perp}$ (perpendicular to the flow velocity $\mathbf{v}_{\mathbf{0}}$ ) on the dependence of the growth rate $\tilde{\gamma}(k d)$ of supersonic longitudinal disturbances (k parallel to the flow velocity $\mathbf{v}_{0}$, or $\mathbf{k}=\mathbf{k}_{\|}$) when $M=2$. Curves 1 and 2 correspond to the following values of the parameter $\beta: \beta=100\left(M_{m s} \approx M=2\right)$ and $\beta=2\left(M_{m s}=1.58\right)$.

The results of calculations presented in Fig. 2 are in agreement with those obtained by Blumen et al. (1975) for the case of $B_{0}=0$ for the values of $M=2$ and $M=1.58$.

Thus, in the case of "hydrodynamic" longitudinal disturbances ( $\mathbf{k}$ parallel to the flow velocity $\mathbf{v}_{0}$ ) the magnetic field $\mathbf{B}_{0}=\mathbf{B}_{0 \perp}$ (perpendicular to the flow velocity $\mathbf{v}_{0}$ ) reduces the compressibility effect of the medium (the value of $M_{m s}$ ) thus exerting a destabilizing influence on the shear flow, increasing the value of a maximum growth rate, and expanding the instability range.

\subsection{Influence of the longitudinal magnetic field}

Let us consider the instability in the domain of sufficiently large values of the "Mach" number $M>1$ in the case of flow along the magnetic field $\mathbf{B}_{0} \| \mathbf{v}_{0}$, where calculations are difficult because of the smallness of the growth rate. Figure 3 is plotted for the following values: $M=2$, and $\beta=2$. We examine the longitudinal (with respect to the flow velocity vector) disturbances $\mathbf{k}=\mathbf{k}_{\|}$.

As has been shown above, the degree of influence of the compressibility depends on the magnetic field strength. Therefore, we shall investigate the influence of Maxwellian tensions (described in Eqs. (4)-(6) by the terms $\propto(\mathbf{k} \cdot \mathbf{a})^{2}=$ $\left.(k a \cos \psi)^{2}\right)$ at a given value of the magnetic field modulus by varying the angle $\psi$ between the magnetic field and the wave vector. In this case the change of the value of $\cos \psi=\mathbf{k} \cdot \mathbf{B}_{0} /\left(k B_{0}\right)$ from 0 to 1 corresponds to the rotation of the vector $\mathbf{B}_{0}$ from the direction normal to the vectors $\mathbf{k}$, and $\mathbf{v}_{0}(\cos \psi=0)$ by the angle $\Delta \psi=\pi / 2$, i.e. to the direction common to $\mathbf{k}$ and $\mathbf{v}_{0}$.

It is evident from the figures that in the case of supersonic disturbances, an increase of $\cos \psi$ is accompanied by a decrease of the maximum value of the growth rate $\tilde{\gamma}_{\max }$. Fur- 


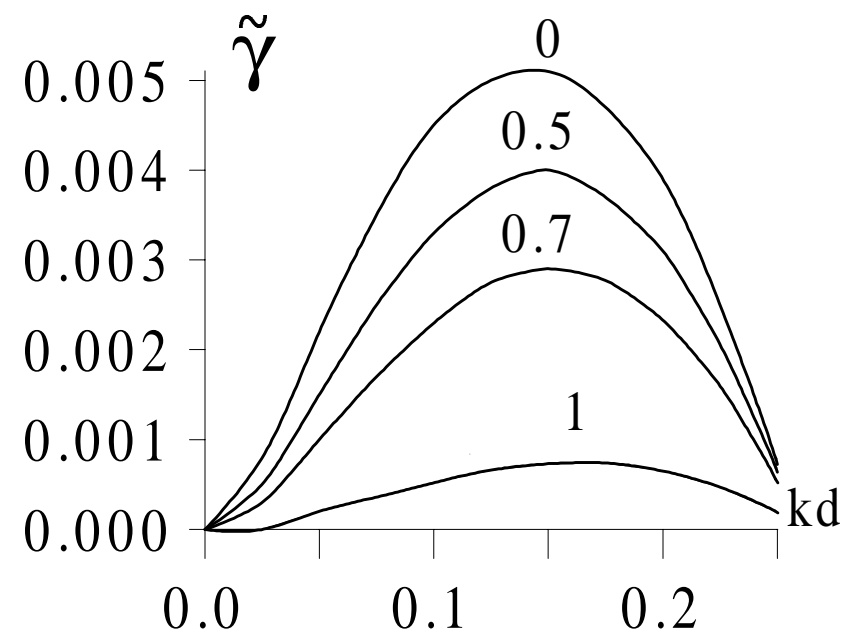

Fig. 3. Influence of the magnetic field on the growth rate of longitudinal ( $\mathbf{k}$ parallel to the flow velocity $\mathbf{v}_{0}, \mathbf{k}=\mathbf{k}_{\|}$) supersonic disturbances $(M=2)$. The value of $\cos \psi=\mathbf{k} \cdot \mathbf{B}_{0} /\left(k B_{0}\right)$ is shown above the curves. "weak field" : $\beta=2, M_{m s}=1.58,-\tilde{\gamma} \neq 0$ when $\cos \psi=1$.

thermore, unlike subsonic disturbances, the instability range does not narrow with an increase of Maxwellian tensions (for values of $\tilde{\gamma} \geq 0.001$ at least).

The position of the short-wavelength boundary of supersonic disturbances is determined by the compressibility level characterized by the value of the "magnetosonic Mach number" $M_{m s}$ which decreases with an enhancement of the field (with a decrease of the parameter $\beta$ ), which causes this boundary to be displaced toward shorter wavelengths. Figure 4 was calculated for the case $\beta=1.2$ when $c_{s}=a$. As our calculations showed, with such a strong magnetic field, the disturbances propagating along the field $\cos \psi=1$ reach total stability $(\tilde{\gamma}=0)$ for all wavelengths. This coincides with a stabilization of the TD with respect to longitudinal disturbances in the case of a flow along the field $c_{s}=a$ (Parker, 1964). At the same time, as is intimated by Fig. 3, no such stabilization occurs when $c_{s}>a(\beta=2)$, i.e. in total agreement with results obtained within the TD approximation (Parker, 1964).

Thus the magnetic field influence on the development of supersonic disturbances is not uniquely manifested. On the one hand, the field increases total pressure thus reducing the compressibility effect. This results in an increase of the growth rate of "hydrodynamic" longitudinal disturbances $(\mathbf{k}$ parallel to flow velocity $\mathbf{v}_{0}$, or $\left.\mathbf{k}=\mathbf{k}_{\|}\right)$in the case $\mathbf{B}_{0}=\mathbf{B}_{0 \perp}$ (perpendicular to flow velocity $\mathbf{v}_{0}$ ) and an expansion of the instability range for all values of $\cos \psi$. Furthermore, the growth rate increases for the flow both in the transverse magnetic field and over a reasonably wide range of values of the angle $\psi:|\cos \psi| \leq 0.7$. Also, the stabilizing effect of Maxwellian tensions still shows up only slightly $\left((\mathbf{k} \cdot \mathbf{a})^{2}\right.$ $\left.\propto(\cos \psi)^{2}\right)$, and hence the field influence manifests itself mainly via an increase in total pressure and a decrease of the value of $M_{m s}$, which leads to an enhancement of the insta-

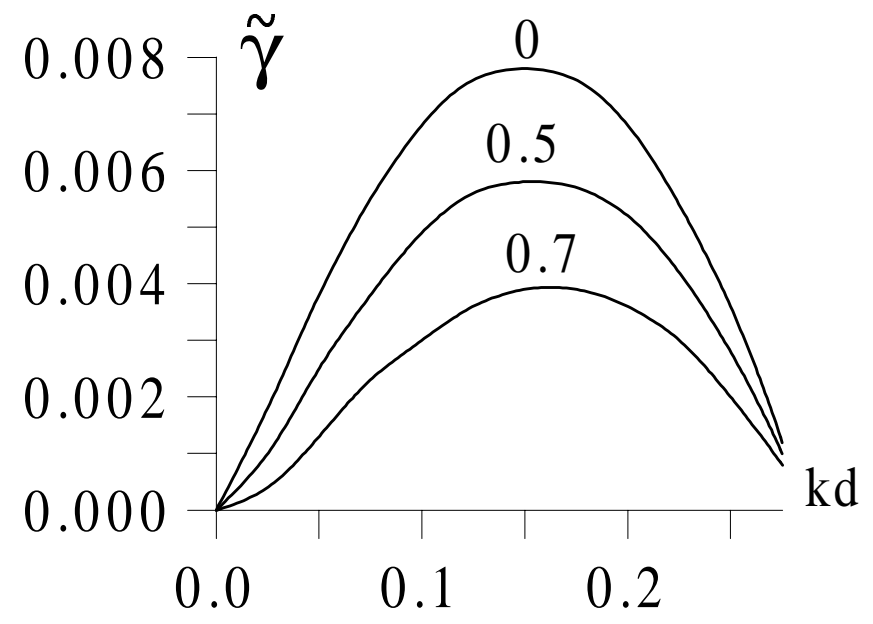

Fig. 4. Influence of the magnetic field on the growth rate of longitudinal (k parallel to the flow velocity $\mathbf{v}_{0}$ ) supersonic disturbances $(M=2)$. "Strong field" : $\beta=1.2$, and $M_{m s}=1.41$; when $\cos \psi=1$ there is stability: $\tilde{\gamma}=0$.

bility. However, at sufficiently large values of the parameter $|\cos \psi|>0.7$, the effect of the stabilizing influence of Maxwellian tensions becomes distinguishable, which when $\cos \psi \rightarrow 1$, i.e. in the case of common direction of $\mathbf{k}, \mathbf{v}_{0}$, and $\mathbf{B}_{0}$, ultimately leads to a total stabilization of longitudinal disturbances when $a \geq c_{s} \quad(\beta \leq 1.2)$.

\section{Role of oblique disturbances in the instability of a supersonic shear flow}

In the foregoing discussion we have considered the longitudinal disturbances (k parallel to flow velocity $\mathbf{v}_{0}$, or $\mathbf{k}=\mathbf{k}_{\|}$) However, within the TD approximation, Syrovatsky (1954) showed that in the case of a supersonic velocity difference, even if the Landau stability criterion (Landau, 1944) is satisfied: $\Delta v \geq 2 \sqrt{2} c_{s}$ (which holds for longitudinal disturbances), the oblique disturbances, for which the wave vector $\mathbf{k}$ in the plane $(x, y)$ is directed at an angle to the velocity vector $\mathbf{v}_{0}$ ), can be unstable. This is because the projection of the flow velocity upon the phase velocity direction can become smaller than the effective sound velocity $c_{m}$, which would lead to a decrease of the wave parameter $\tilde{M}^{2}=\left(\omega-\mathbf{k} \cdot \mathbf{v}_{0}(z)\right)^{2} /\left(k c_{m}\right)^{2}=\left(\tilde{\omega} /\left(k c_{m}\right)\right)^{2}$ and, accordingly, to an increase of the growth rate (see also the discussion of the role of three-dimensional disturbances in (Dunn and Lin, 1952; Fejer and Miles, 1963).

In spite of the fact that in the hydrodynamics the importance of oblique disturbances is, in principle, known (Blumen et al., 1975; Goldstein and Choi, 1989), it is customary to neglect their role in analyses of the instability of particular supersonic shear flows in geospace plasma. For that reason, it was of interest to us to prove our conclusions about the predominance of oblique disturbances using supersonic shear 


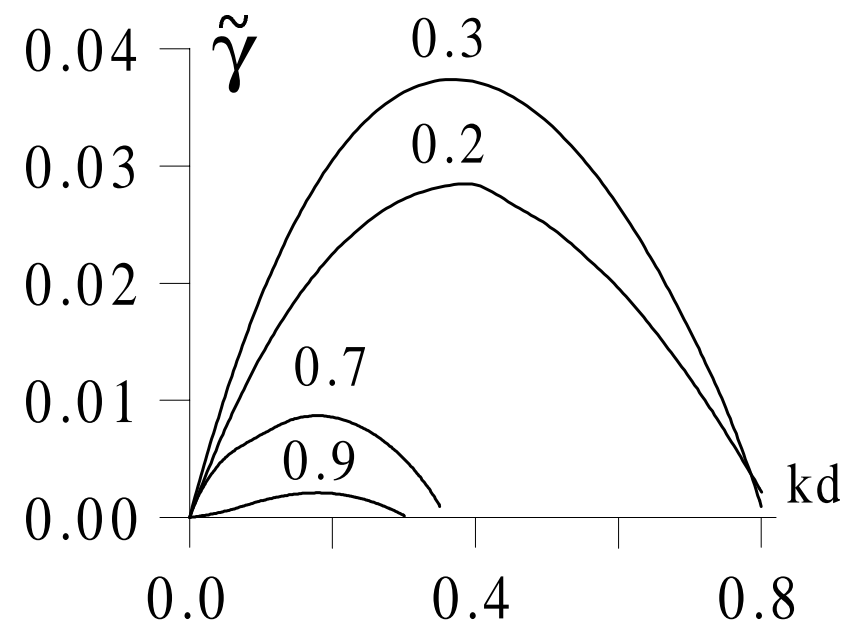

Fig. 5. Influence of $\cos \phi$ on the dependence of the growth rate $\tilde{\gamma}(k d)$ for the magnetic field aligned with the flow $\mathbf{v}_{0}$, or $\mathbf{B}_{0}=\mathbf{B}_{0 \|}$ when $M=2, \beta=1.5, M_{m s}=1.49$, and $\rho=$ const . The value of $\cos \phi$ is shown above the curves.

flows at the magnetospheric boundary (Mishin and Morozov, 1983) and in the solar wind (Korzhov et al., 1984).

The influence of the inclination angle $\phi$ of the wave vector with respect to the flow velocity direction $(\cos \phi=$ $\left.\left(\mathbf{k} \cdot \mathbf{v}_{0}\right) /\left(k v_{0}\right)\right)$ is shown in Fig. 5. Consider the case of the magnetic field aligned with the flow, or $\mathbf{B}_{0}=\mathbf{B}_{0 \| \mid}$, where the stabilizing effect of Maxwellian tensions is maximal.

For the parameters selected, the magnetosonic Mach number $M_{m s}=1.49\left(M_{a}=\sqrt{5}\right)$. Since the phase velocity $v_{p h} \propto \cos \phi$, the "Mach wave" number $\tilde{M}=\tilde{\omega} /\left(k c_{m}\right)$ obeys the relationship: $\tilde{M} \propto \cos \phi$ (see Squire's transformation in Blumen, 1970). Hence a decrease of $\cos \phi$ is accompanied by a decrease of $\tilde{M}$, and when $\cos \phi \leq 0.7$ there occurs a transition to subsonic disturbances $\tilde{M} \leq 1$. This implies not only an increase of the growth rate but also an abrupt decrease of the imaginary part of the coefficient $\chi$, as well as a significant displacement of the boundary $\alpha_{b}$ toward shorter wavelengths.

Besides, additional calculations showed that in the case of a change of the angle $\psi$ between the magnetic field and the wavevector $\mathbf{k}$, the pair of the upper curves and the pair of the lower curves show a difference in the behavior of the short-wavelength boundary. Thus, for subsonic disturbances (upper curves), with an increase of $\cos \psi$ (with an increase of Maxwellian tensions), the boundary $\alpha_{b}$ is displaced to the left - in accordance with results reported by Chandra (1973) and obtained within the incompressibility approximation. For supersonic disturbances (lower curves), however, the value of $\alpha_{b}$ will remain unchanged (cf. Fig. 3).

Since the decrease of $\cos \phi$ is accompanied by a decrease of the influence not only of the compressibility of the medium but also of Maxwellian tensions $(\cos \psi=\cos \phi)$, the dependence $\tilde{\gamma}(\phi)$ at a given $|\mathbf{k}|$ must have a maximum. In the case under consideration, subsonic disturbances undergo the most intense excitation when $\cos \phi \approx 0.3-0.4$.
Thus the most dramatic (in our opinion) manifestation of the influence of the compressibility of the medium involves the predominance of slow oblique disturbances when compared with supersonic longitudinal disturbances, both in regard to the instability range (when $M_{m s} \cos \phi<1$ it expands sharply to $\alpha_{b} \geq 0.8$ ), and in the value of the growth rate $\tilde{\gamma}_{\max }$.

\section{Instability at the magnetospheric tail boundary}

We now examine the instability having regard to the density and magnetic field inhomogeneities, as well as the supersonic velocity difference at the geomagnetic tail boundary for a set of parameters obtained by the ISEE-3 satellite in the distant tail region $\left(-220 R_{E}<x<-50 R_{E}\right)$ (i.e. for the set of parameters analyzed in Mishin and Morozov, 1983).

According to the data reported in (Bame et al., 1983), in the magnetosheath (region II) we have $n_{I I}=8.5 \mathrm{~cm}^{-3}$, $T_{I I}=2 \cdot 10^{5} \mathrm{~K}$, and $v_{I I}=500 \mathrm{~km} / \mathrm{s}$, while in region I (plasma mantle or outer region of the plasma sheet, or low-latitude boundary layer), $n_{I}=1 \mathrm{~cm}^{-3}$, and $T_{I}=$ $(3-10) \cdot 10^{5} \mathrm{~K}$. By specifying the value of the magnetic field $B_{I}$ for one side of the boundary, it is easy to determine its value for the other side from the balance condition of thermal and magnetic pressures. Thus for $B_{I I}=6 \mathrm{nT}$ we obtain $B_{I}=9 \mathrm{nT}$.

Plasma flow in the high-latitude boundary layer of the geomagnetic tail is aligned with geomagnetic field lines $\left(\mathbf{B}_{0}=\right.$ $\left.\mathbf{B}_{0 \|}\right)$ whereas in the magnetosheath the magnetic field can also have a component $\mathbf{B}_{0}=\mathbf{B}_{0 \perp}$ (perpendicular to the flow velocity $\mathbf{v}_{0}$ ). Using ISEE-3 data we calculated the dependence $\tilde{\gamma}(k d)$ in the least favorable (for the development of an instability) magnetic field aligned with the flow $\mathbf{v}_{0}$, or $\mathbf{B}_{0}=\mathbf{B}_{0 \|}$ for the following (describing quite well the ISEE3 observations) parameters, $\varrho=3.75, \rho(z=0)=\rho_{00}=$ $4.75, \delta=1 / 6, \beta_{I}=0.125, \beta_{00}=\beta(z=0)=0.65$, and $\beta_{I I}=1.65$.

\subsection{Instability as a function of the velocity difference}

Calculations were performed for three possible values of the velocity difference at the boundary: $1-\Delta v=v_{I I} / 2=$ $250 \mathrm{~km} / \mathrm{s}, \quad 2-\Delta v=335 \mathrm{~km} / \mathrm{s}$, and $3-\Delta v=v_{I I}=$ $500 \mathrm{~km} / \mathrm{s}$. The point here is that the flow velocity can be lower than the solar wind velocity at the boundary of the "near" tail $\left(x>-50 R_{E}\right)$. Besides, a velocity difference can be taking place in two stages: initially, a dramatic difference at the outer edge of the boundary layer, and then a smoother difference inside the edge. Figure 6 shows the influence of the change of the cosine of the angle $\phi$ (shown above the curve) between the wave vector and the velocity vector for the value of $M_{00}=M(z=0)=2.24$, i.e. for the velocity difference $\Delta v=2 u=v_{I I} / 2=250 \mathrm{~km} / \mathrm{s}$ at the boundary. As is evident, even with such a relatively small velocity difference, there arises an instability with a sufficiently significant growth rate $\tilde{\gamma} \leq 0.02$. 


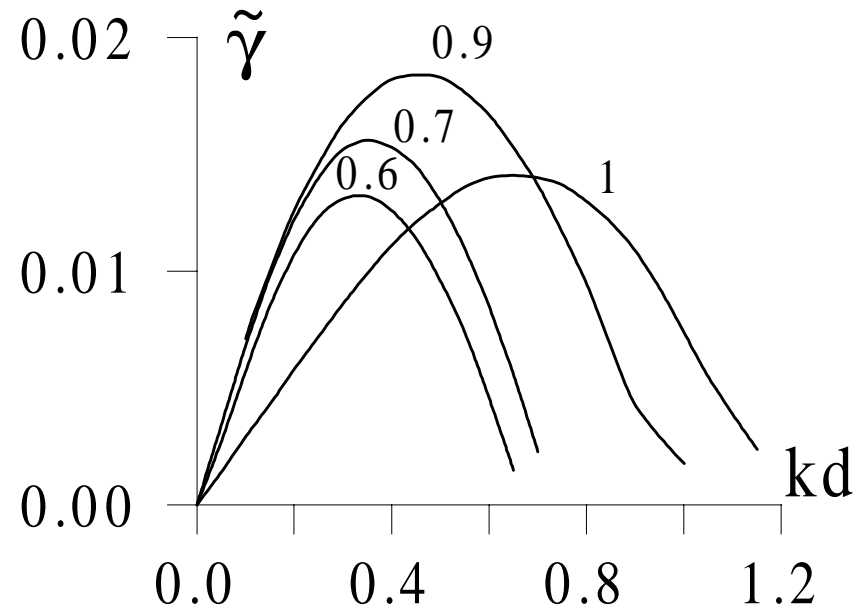

Fig. 6. Instability of the high-latitude boundary layer of the geomagnetic tail for the velocity difference $\Delta v=250 \mathrm{~km} / \mathrm{s} \quad\left(M_{00}=\right.$ 2.24) as a function of $\cos \phi$. The case of the magnetic field aligned with the flow $\mathbf{v}_{0}$.

Note that using the TD approximation McKenzie (1970) and Ershkovich and Nusinov (1972) obtained a more stringent condition for K-H instability development at the tail boundary: $\Delta v>400 \mathrm{~km} / \mathrm{s}$. Satellite data (Sibeck et al., 1987) show, however, the presence of tail boundary oscillations when $\Delta v \geq 250 \mathrm{~km} / \mathrm{s}$. This does confirm the results presented in Fig. 5. A strong longitudinal magnetic field, in addition to having a stabilizing effect of Maxwellian tensions, reduces significantly the influence of the compressibility: when $\beta_{00}=0.65$ we have $M_{m s}(z=0)=1.33$. In fact, however, the "effective" value of the Mach wave number $<\tilde{M}>\sim 1$, because the ratio $\left|\chi_{i m} / \chi_{\text {re }}\right|$ is large only for longitudinal disturbances $\cos \phi=1$, but already when $\cos \phi \leq 0.9$ the phase velocity becomes subsonic, and the disturbances become surface disturbances $\left|\chi_{i m} / \chi_{\text {re }}\right| \leq 1$. The large values of $M_{m s}$ are caused by a strong velocity difference.

It seems likely that because of the large field strength $\left(M_{a 00}=M_{a}(z=0)=1.65\right)$, with an increase of the angle $\phi$, there occurs a narrowing of the instability range, i.e. a decrease of the value of $\alpha_{b}$. Thus, if we remove the stabilizing influence of Maxwellian tensions by putting $\cos \psi=\mathbf{k} \cdot \mathbf{B}_{0} /\left(k B_{0}\right)=0$, then (see Fig. 7), in addition to an increase of a maximum growth rate by about a factor of 4 , we can see a displacement of the instability boundary toward shorter-wavelengths $\alpha_{b}$ with an increase of the angle $\phi$ and with a corresponding decrease of $M_{m s}$, similar to what is taking place when $\beta>1$ and $\rho=$ const. Such an instability enhancement must occur at the low-latitude boundary layer of the tail where there is a significant component of the geomagnetic field perpendicular to the flow direction.

As the velocity difference at the boundary increases, the compressibility influence is enhanced, and the influence of Maxwellian tensions decreases. Figure 8 illustrates the case $M_{00}=3$, and $M_{a 00}=2.2$ corresponding to the value of

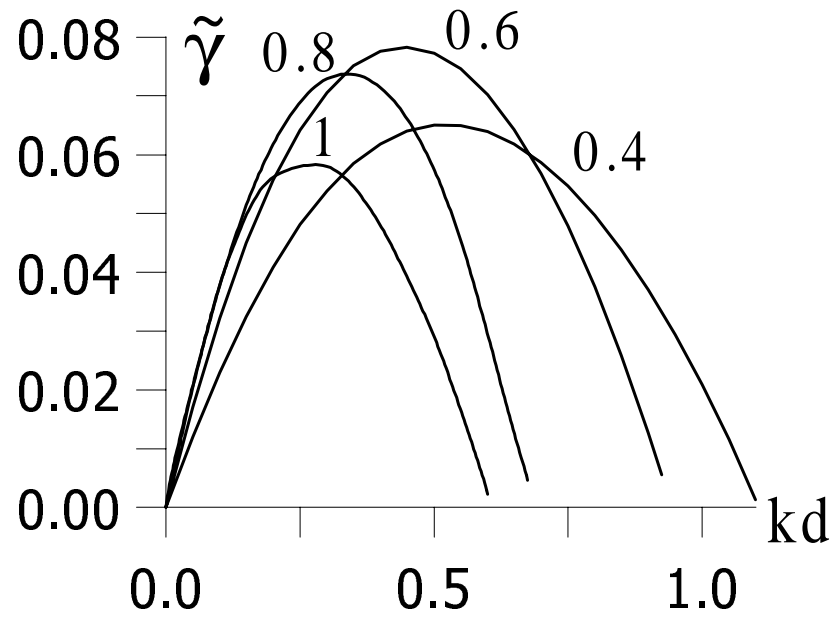

Fig. 7. Instability of the geomagnetic tail boundary layer for the velocity difference $\Delta v=250 \mathrm{~km} / \mathrm{s}\left(M_{00}=2.24\right)$ as a function of $\cos \phi$. The case of the absence of Maxwellian tensions: $\mathbf{B}_{0} \perp \mathbf{k}$.

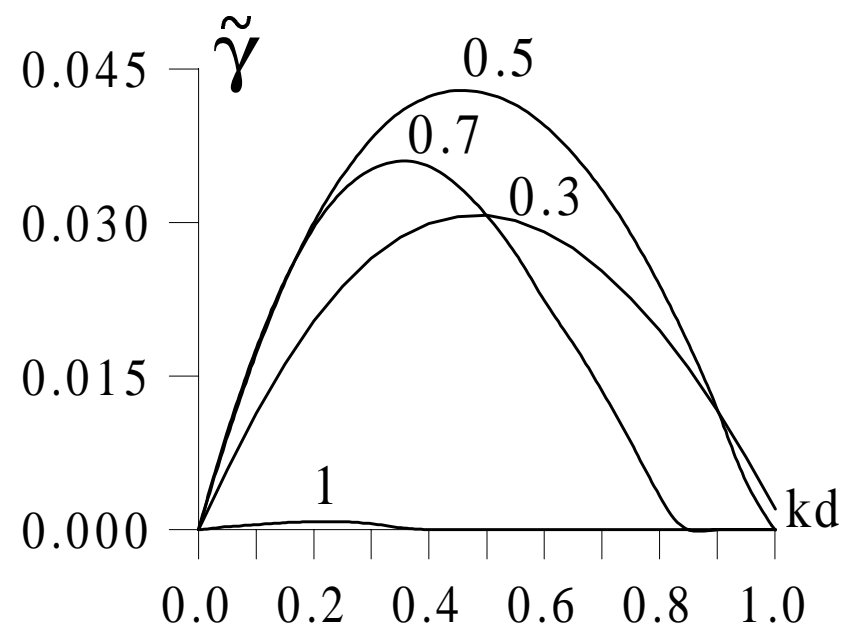

Fig. 8. Instability of the boundary layer of the distant tail for the velocity difference $\Delta v=335 \mathrm{~km} / \mathrm{s}\left(M_{00}=3\right)$ as a function of $\cos \phi$. The case of the magnetic field aligned with the flow $\mathbf{v}_{0}$.

the velocity difference, $\Delta v=335 \mathrm{~km} / \mathrm{s}$. With these parameters, the longitudinal field influence is markedly weak, and the character of the influence of the angle $\phi$ is the same as in the absence of Maxwellian tensions in the preceding figure. At the same time, an increase of the number $M$ to 3 (see Fig. 8) manifests itself in the decrease of the growth rate of longitudinal $(\cos \phi \leq 1)$ disturbances and in the expansion of the opening of the angle $\phi$ of generation of supersonic disturbances - the transition to surface disturbances is taking place when $\cos \phi \leq 0.7$.

In the case of a maximum possible velocity difference at the boundary $\Delta v=v_{I I}=500 \mathrm{~km} / \mathrm{s}$, we have an enhancement of the compressibility influence, which is manifested in the generation of supersonic disturbances over a wider range of values of the angle $\phi(\cos \phi \leq 0.5)$, but with smaller growth rates as a consequence of an increase of the magne- 


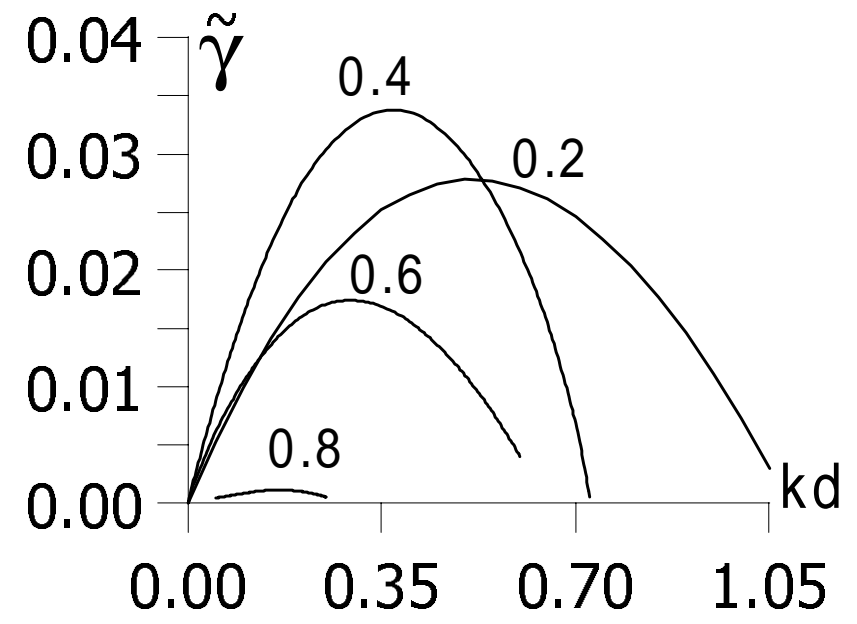

Fig. 9. Instability of the boundary layer of the distant tail for the velocity difference $\Delta v=500 \mathrm{~km} / \mathrm{s}\left(M_{00}=4.5\right)$ as a function of $\cos \phi$. The case of the magnetic field aligned with the flow $\mathbf{v}_{0}$.

tosonic Mach number: $M_{m s 00} \approx 2.6$. In this case the role of the Maxwellian tension becomes weak because of the large value of the Alfven Mach number $\left(M_{a 00} \approx 3.3\right)$. Note that within the approximation of a weak compressible medium the stabilizing influence of Maxwellian tensions is stronger for $M_{a 00}=\sqrt{2}$ than for $M_{a 00}=2$.

At this point, it is necessary to remark that in all possible cases of the instability development, the destabilizing role is played by the density inhomogeneity. This is easy to see from the expansion of the instability range: the position of the short-wavelength boundary of subsonic oblique disturbances in Figs. 7 and 9 corresponds to the value of $\alpha_{b} \geq 1-$ i.e. it lies father from the boundary of subsonic disturbances in the case of subsonic disturbances in the case of a uniform density. The density inhomogeneity influence implies a decrease of the phase velocity of the disturbances generated (see below) and, hence, a reduction of the compressibility influence.

Thus we have demonstrated that the distant tail boundary for actually observed parameters of plasma and the magnetic field is unstable at all latitudes; furthermore, the influence of the longitudinal magnetic field (aligned with the flow) is almost indistinguishable when the velocity difference $\Delta v \geq$ $335 \mathrm{~km} / \mathrm{s}$.

5.2 On the frequency and wavelength of the generated oscillations

In the last case $\Delta v=500 \mathrm{~km} / \mathrm{s}$ for oblique disturbances with a maximum growth rate when $\cos \phi=0.4$ we have $\omega=0.2 v_{I I} / D, \alpha_{\max }=0.35, v_{\text {phmax }}=0.28 v_{I I}$.

Note that the phase velocity changes little over the entire instability range: when $\alpha=0.05$ we have $v_{p h} \approx 0.3 v_{I I}$, and when $\alpha=0.7$ we have $v_{p h} \approx 0.265 v_{I I}$, so that the value $v_{\text {phmax }}$ virtually coincides with the mean value of the phase velocity over the entire instability range. For the sake of comparison, we must point out that within the TD approximation the phase velocity of longitudinal disturbances is much larger: $v_{p h} \approx 0.9 v_{I I}$. Therefore, longitudinal longwavelength disturbances are virtually suppressed by a strong compressibility.

Let us estimate the period of the most unstable disturbances. For the typical value of the high-latitude boundary layer thickness of the distant tail $D=10^{4} \mathrm{~km}$, we obtained $T \geq 10 \mathrm{~min}$, which is in good agreement with ISEE-3 observations of long-period oscillations of the distant tail boundary in the range of periods of 10-40 min (Sibeck et al., 1987). The wavelength corresponding to a maximum growth rate, is $\lambda=v_{\text {phmax }} \cdot T \leq 10^{5} \mathrm{~km} \approx 15 R_{E}$. To interpret the ISEE-3 crossings of the distant tail boundary in (Sibeck et al., 1987) when calculating the magnetopause oscillation wavelength, it was assumed that the value of the wave propagation velocity $v_{p h}=v_{I I}$, which is nearly by a factor of 4 larger than the value of the phase velocity which we obtained. As a result, a too high estimate of the magnetopause oscillation wavelength was obtained: $\lambda>100 R_{E}$, which led Sibeck et al. (1987) to incomprehension of how such long waves can be excited at the magnetopause. To explain this far-fetched "paradox", Belmont and Chanteur (1989) chose to look for the possible nonlinear transformation of the waves into the long-wavelength region (see also Miura, 1999), which, as we see, was in fact not needed (but gave interesting results).

The distant tail boundary instability can be the source of long-period ( $T \geq 10 \mathrm{~min}$ ) geomagnetic pulsations Pc6 observed in the night-time at high latitudes (Pudovkin et al., 1976), i.e. near the ground projection of the geomagnetic tail boundary.

\section{Discussion}

First we discuss some of the assumptions made in this paper. We have used the MHD approximation and ignored the role of kinetic effects. Line-tying and kinetic effects can influence the conditions of instability development on the dayside and near the dawn-dusk meridian. However, this influence is small in the far tail. Thus the line-tying effect can have a stabilizing influence on the instability in the presence of a field-aligned current. This influence can be substantial when the Alfven transit time $\tau_{a}$ between two ionospheres is comparable to or smaller than the growth (e-folding) time $\left(\gamma^{-1}\right)$ of the K-H instability (Miura, 1996). For the most unstable disturbances at the far tail boundary we have $x=-100 R_{E}$, and $d=R_{E}$, i.e. an inverse inequality $\gamma^{-1} / \tau_{a} \ll 1$ holds.

Next, as regards the influence of taking into account the kinetic effects. It is known that accounting for the finite Larmor radius is substantial for the dayside magnetopause because the thickness of the shear layer there is on the order of several Larmor radii (Nagano, 1978). However, in the far tail the thickness of the shear layer is much larger, $d \approx R_{E}$, and the value of the proton gyroradius for the parameters used in the paper is $\rho_{L} \approx 200 \mathrm{~km}$. For the most unstable disturbances $\left(k d=k R_{E} \approx 0.5\right)$, the contribution of the kinertic 
effects is small, $k \rho_{L} \approx 1 / 60$. Thus, their role (as well as of the line-tying effect) for the instability development at the far tail boundary can be safely neglected.

For the sake of simplicity, our calculations use an identical scale of variation of all plasma and magnetic field parameters on the shear layer. In effect the field usually changes on a smaller scale, and this occurs in the outer part of the boundary layer, the magnetopause. Therefore, specifying identical scales enhances the role of the external field (magnetosheath field) and attenuates the role of the geomagnetic field in the layer of a thickness determined by the difference of the scales of variation of the velocity and the field. In the case of a longitudinal flow at the far tail boundary, this leads to an enhancement of the stabilizing influence of the field-aligned geomagnetic field as well as to a decrease of the instability growth rate. Consequently, for the real profile of the magnetic field variation, the value of the growth rate will be larger. Thus, the values obtained in this paper are the lower estimates of the growth rate value.

In this paper we neglect the rotation of the magnetic vector on the shear layer. However, when analyzing the instability of the geomagnetic tail boundary, we examine the most unfavorable case of the instability development where the field is parallel to the flow velocity throughout the entire volume. Therefore, taking into account the rotation of the field implies an attenuation of its stabilizing influence and, hence, an increase of the growth rate.

We now describe quantitatively the compressibility influence of the medium when the magnetic field, the density inhomogeneity, and the angle of inclination of the wave vector with respect to the velocity vector of the medium are taken into account. In the system of Eqs. (4)-(5), this influence is described by the the function $\chi$ characterizing the exponential decrease of disturbances. In the absence of this influence (i.e. when $M=0$, and $\operatorname{div} \mathbf{v}=0$ ), this term becomes zero. Furthermore, the index of decrease of the disturbance amplitude is maximal, $\chi=\operatorname{Re}(\chi)=k$, and $\operatorname{Im}(\chi)=0$. In the case of a finite compressibility of the medium (when $M \neq 0$, and $\operatorname{div} \mathbf{v} \neq 0$ ), the coefficient of exponential decay decreases because of the appearance of its imaginary part and, accordingly, the decrease of the real part. The Mach wave number $\tilde{M}=\tilde{\omega} /\left(k c_{m}\right)$ must be taken as the measure of compressibility influence. Since the "shifted" frequency $\tilde{\omega}$ is proportional to the scalar product $\tilde{M} \propto \mathbf{k} \cdot \mathbf{v}$ (i.e. also to the cosine of the angle between these vectors), the Mach wave number is proportional to the flow velocity projected onto the direction of the wave vector: $\tilde{M} \propto u \cdot \cos \phi / c_{m} \propto M_{m s} \cdot \cos \phi$. Here the proportionality coefficient is determined by the difference of the values of the phase velocity and the local (as a function of coordinate $z$ ) flow velocity: $\tilde{\omega} / k=(\omega / k-v(z) \cdot \cos \phi)$. Within the TD approximation which holds for long-wavelength disturbances, the value of the velocity is determined by the "mean-mass" velocity: $\omega=\mathbf{k} \cdot\left((n \mathbf{v})_{I}+(n \mathbf{v})_{I I}\right) /\left(n_{I}+n_{I I}\right)$ (Mishin, 1981). With the density difference $n_{I I} / n_{I} \gg 1$ existing at the magnetospheric boundary, the phase velocity is determined by the flow velocity projected onto the direction of the wave vector: $\omega / k \leq v_{I I} \cos \phi$. As a result, for long-wavelength waves we obtain an estimate of the Mach wave number in the magnetosheath (for the density values used in this paper): $\tilde{M}_{I I}=\tilde{\omega} /\left(k c_{m I I}\right) \leq 0.1 M_{m s I I} \cos \phi$. Thus outside of the magnetosphere, the value of the Mach wave number, in view of the difference $\tilde{\omega} / k=(\omega / k-v(z) \cdot \cos \phi)$, is significantly smaller than the value of the magnetosonic Mach number, which is caused, as has been shown above, by the density inhomogeneity. Also, inside of the magnetosphere the value of the magnetosonic Mach number is smaller than that outside of it because of the large value of the magnetosonic velocity there. Consequently, the density inhomogeneity reduces the compressibility influence of the medium of the outer halfspace (magnetosheath) where this influence is the strongest.

Thus the use of the Mach wave number $\tilde{M}$ makes it possible to mathematically describe the attenuation of the compressibility influence of the medium through the following three factors: (1) the increase of total pressure by magnetic pressure through a decrease of the magnetosonic Mach number (see Fig. 2), (2) a strong density increase in the magnetosheath, and (3) taking into account the oblique disturbances $\cos \phi \neq 0$. That is why at the boundary of the far tail we obtain such an effective excitation of oblique disturbances, in spite of the large values of the Mach number.

These results can be used in the analysis of the saturation level of nonlinear development of the instability. Thus Miura (1990, 1992), based on a numerical simulation, obtained a dramatic decrease of the oscillation amplitude in the case of supersonic disturbances. In doing so, he addressed the 2D problem, namely, he only considered longitude disturbances $\left(\mathbf{k}=\mathbf{k}_{\|}\right)$. If one takes into consideration that the oblique disturbances satisfy the Squire transform (Blumen et al., 1975), one can calculate the amplitude of steady-state oscillations caused by transonic oblique disturbances on a supersonic shear flow. It is found in this case that the linear growth rate of oblique disturbances for $M=1.5-2$ is by a factor of two to four smaller than that of longitudinal ones for $M=1$. For this reason, the saturation level at the far tail boundary will also be only several times smaller when compared with $M=1$ (rather than by one or two orders of magnitude as determined by Miura, 1992) for longitudinal disturbances.

We intend to present the specific results derived from calculating the influence of nonlinear effects elsewhere.

\section{Conclusions}

By analyzing the supersonic shear flow instability on the geomagnetic tail boundary, we have demonstrated the validity of the conclusions drawn by Mishin and Morozov (1983) about the predominant role of three-dimensional or oblique (with respect to the direction of the velocity vector) disturbances. Their phase velocity is significantly smaller (1/4 of the flow velocity), and the value of the growth rate and the width of the frequency range are significantly larger than those for two-dimensional (longitudinal) disturbances. Furthermore, the value of the dimensionless growth rate of the most un- 
stable oblique disturbances are almost identical to those calculated in Mishin and Morozov (1983), i.e. they are of the same order of magnitude as in the case of a near-sonic velocity difference.

The magnetic field and the density inhomogeneity attenuate the stabilizing influence of the compressibility of the medium, which leads to an expansion of the instability range and to an increase of the growth rate over a wide range of values of the angle $\phi$.

Thus we have confirmed the conclusions from Mishin and Morozov (1983) that, in spite of the hypersonic character of the flow around the distant geomagnetic tail, near-sonic oblique disturbances can play a substantial role in the excitation of MHD waves and in the evolution of the tail boundary. This applies also to the conclusions drawn in (Korzhov et al., 1984) about the instability of shear flows in the solar wind where the generation of oblique disturbances accounts for the spectrum of the waves observed there, and for the presence of a wide shear layer (Goldstein, 1995).

Acknowledgements. The author wishes to acknowledge helpful discussions with I. G. Shukhman. This work was supported by RFBR grants 01-05-64203 and 02-05-39020.

\section{References}

Bame, S. J., Anderson, R. C., Asbridge, J. R., Baker, D. N., Feldman, W. C., Gosling, J. T., Hones, E. W., McComas, D. J., and Zwickl, R. D.: Plasma regimes in deep geomagnetic tail: ISEE 3, Geophys. Res. Let., 10, 912-915, 1983.

Belmont, G. and Chanteur, G.: Advances in magnetopause KelvinHelmholtz instability studies, Phys. Scr., 40, 124-128, 1989.

Blumen, W.: Shear layer instability of an inviscid compressible fluid, J. Fluid Mech., 46, 763-775, 1970.

Blumen, W., Drazin, P. G., and Billings, D. F.: Shear layer instability of a inviscid compressible fluid, Part 2, J. Fluid Mech., 71, 305-316, 1975.

Boller, B. R. and Stolov, H. L.: Kelvin-Helmholtz instability and the semiannual variation of geomagnetic activity. J. Geophys. Res., 75, 6073-6084, 1970.

Chandra, K.: Hydromagnetic stability of plane heterogeneous shear flow. J. Phys. Soc. Japan, 34, 539-546, 1973.

Chandrasekhar, S.: Hydrodynamic and hydromagnetic stability. Clarendon Press, Oxford, 1962.

Drazin, P. G. and Davey, A.: Shear layer instability of an inviscid compressible fluid, Part 3, J. Fluid Mech., 82, 255-260, 1977.

Dungey, J. W.: Electrodydamics of the outer atmosphere, Proceedings of the Ionosphere, 225, Phys. Society of London, London, 1955.

Dunn, D. W. and Lin, C. C.: The stability of the laminar boundary layer in a compressible fluid for the case of three-dimensional disturbances, J. Aero. Sci., 19, 491, 1952.

Ershkovich, A. I. and Nusinov, A. A.: Geomagnetic tail oscillations, Cosmic. Electrodynamics, 2, 471-490, 1972.

Farrugia C. J., Gratton F. T., Contin J., Cocheci C. C., Arnoldy, R. L., Ogilvie, K. W., Lepping, R. P., Zastenker, G. N., Nozdrachev, M. N., Fedorov, A., Sauvaud, J.-A., Steinberg, J. T., and Rostoker, G.: Coordinated Wind, Interball/tail, and ground observations of Kelvin-Helmgoltz waves at the near-tail, equatorial magnetopause at dusk: 11 January 1997, J. Geophys. Res., 105, 7639-7667, 2000.

Fejer, J. A. and Miles, J. W.: On the stability of a plane vortex sheet with respect to three-dimensional disturbances, J. Fluid Mech., 15, 336-336, 1963.

Goldstein, M. 1., Roberts, D. A., and Matthaeus, W. H.: Magnetohydrodynamic turbulence in the solar wind, Annual Rev. Astron. Astrophys., 33, 283-325, 1995.

Goldstein, M. E. and Choi, S.-W.: Nonlinear evolution of interacting oblique waves on two-dimensional shear layers, J. Fluid. Mech., 207, 97-120, 1989.

Kivelson, M. G. and Chen, S.-H.: The magnetopause: surface waves and instabilities and their possible dynamical consequences, Geophys. Monograph 90, Physics of the magnetopause, (Eds) Song, P., Sonnerup, B. U. O., and Thompsen, M. P., 257268, 1995.

Korzhov, N. P., Mishin, V. V., and Tomozov V. M.: On the role of plasma parameters and the Kelvin-Helmholtz instability in viscous interaction of solar wind streams. Planet. Space Sci., 32, 1169-1178, 1984.

Kovner, M. S., Mishin, V. V., and Skelev, E. I.: On hydromagnetic pulsations in the magnetosphere and the Kelvin-Helmholtz instability, Sov. Phys.-Geomagnetizm i aeronomiya, 17, 714-718, 1977.

Landau L. D.: Stability of tangential discontinuities in compressible fluid, Akad. Nauk S.S.S.R., Compts Rendus (Doklady). 44, 139_ 142, 1944.

Mann, I. R., Wright, A. N., Mills, K., and Nakariakov V. M.: Excitation of magnetospheric waveguide modes by magnetosheath flows, J. Geophys. Res., 104, 333-353, 1999.

McKenzie, J. F.: Hydromagnetic wave interaction with the magnetopause and the bow shock, Planet. Space Sci., 18, 1-23, 1970.

Michalke, A.: On the inviscid instability of the hyperbolic tangent profile, J. Fluid. Mech, 19, 689-702, 1964.

Mishin, V. V.: On the viscous interaction of the solar wind with the magnetosphere and the Kelvin-Helmholtz instability, Sov. Phys.Geomagnetizm i aeronomiya, 19, 943-945, 1979.

Mishin, V. V.: On the MHD instability of the Earth's magnetopause and its geophysical effects, Planet. Space Sci., 29, 359-363, 1981.

Mishin, V. V. and Morozov, A. G.: On the effect of oblique disturbances on Kelvin-Helmholtz instability at magnetospheric boundary layers and in solar wind, Planet. Space Sci., 31, 821828, 1983.

Miura, A. and Pritchett, P. 1.: Nonlocal analysis of the KelvinHelmholtz instability in compressible plasma, J. Geophys. Res., 87, 7431-7444, 1982.

Miura, A., Kelvin-Helmholtz instability for supersonic shear flow at the magnetospheric boundary, Geophys. Res. Lett., 17, 749752, 1990.

Miura, A.: Kelvin-Helmholtz instability at the magnetospheric boundary: dependence on the magnetosheath sonic Mach number, J. Geophys. Res., 97, 10 655-10 675, 1992.

Miura, A.: Stabilization of the Kelvin-Helmholtz instability by the transverse magnetic field in the magnetosphere-ionosphere coupling system, Geophys. Res. Lett., 23, 761-764, 1996.

Miura, A.: Self-organization in the two-dimensional magnetohydrodynamic transverse Kelvin-Helmholtz instability, J. Geophys. Res., 104, 395-411, 1999.

Morozov, A. G. and Mishin, V. V.: Influence of the magnetospheric boundary layer structure on Kelvin-Helmholtz instability, Sov. Phys.-Geomagnetizm i aeronomiya, 21, 1044-1049, 1981. 
Moskvin, Yu. L. and Frank-Kamenetsky, D. A.: Geomagnetic micropulsations, and the solar wind, Sov. Phys.-Geomagnetizm i aeronomiya, 7, 144-149, 1967.

Nagano, H.: Effect of finite ion Larmor radius on the KelvinHelmholtz instability, J. Plasma Phys., 20, 149-160, 1978.

Ong, R. S. B. and Roderick, N.: On the Kelvin-Helmholtz instability of the Earth's magnetopause, Planet. Space Sci., 20, 1-9, 1972.

Parker, E. N.: Dynamical properties of stellar coronae and stellar winds, Astrophys. J., 139, 690-709, 1964.

$\mathrm{Pu}, \mathrm{Z}$. Y. and Kivelson, M. G.: Kelvin-Helmholtz instability at the magnetopause, J. Geophys. Res., 88, 841-852, 1983.

Pudovkin, M. I., Raspopov, O. A., and Kleimenova, N. G.: Disturbances of the Earth's Electromagnetic Field, Pt. 2. Short-Period Oscillations of the Geomagnetic Field, Leningrad: Izd-vo LGU, 1976.

Rayleigh, J. W. S.: The theory of sound. 2, Chap. XXI, Dover, New York, 1945, reprint of second edition 1894.

Sen, A. K.: Effects of compressibility on the Kelvin-Helmholtz instability in plasma, Phys. Fluids, 7, 1293-1298, 1964.

Shen, C. and Liu, Z. X.: The coupling mode between Kelvin-
Helmholtz and resistive instabilities in compressible plasmas, Phys. Plasmas, 6, 2883-2889, 1999.

Shukhman, I. G.: Nonlinear evolution of a weakly unstable wave in a a free shear flow with a weak parallel magnetic field, J. Fluid. Mech., 369, 217-252, 1998.

Sibeck, D. G., Slavin, J. A., and Smith, E. J.: ISEE-3 magnetopause crossings: evidence for the Kelvin-Helmholtz instability, Magnetotail physics, edited by Lui, T. Yz., 73-76, J. Hopkins Univ. Press, Baltimore, 1987.

Southwood, D. J.: Some features of field line resonances in the magnetosphere, Planet. Space Sci., 22, 483-489, 1974.

Southwood, D. J.: Magnetopause Kelvin-Helmholtz instability, Proceedings of the Chapman conference on magnetospheric boundary layers, Eur. Space Agency Spec. Publ., ESA SP, 148, 357-371, 1979.

Syrovatsky, S. I.: Instability of tangential discontinuities in a compressible medium, Sov. Phys.-JETP, 27, 121-123, 1954.

Walker, A. D. M.: The Kelvin-Helmholtz instability in the lowlatitude boundary layer, Planet. Space Sci., 29, 1119-1133, 1981

Wu, C. C.: Kelvin-Helmholtz instability at the magnetopause boundary, J. Geophys. Res., 91, 3042-3059, 1986. 